\title{
Static and dynamic correlation lengths in supercooled polymers
}

Cite as: J. Chem. Phys. 150, 234508 (2019); https://doi.org/10.1063/1.5091682

Submitted: 04 February 2019 . Accepted: 31 May 2019 . Published Online: 21 June 2019

Cristian Balbuena, Melisa M. Gianetti, and Ezequiel R. Soulé (iD)
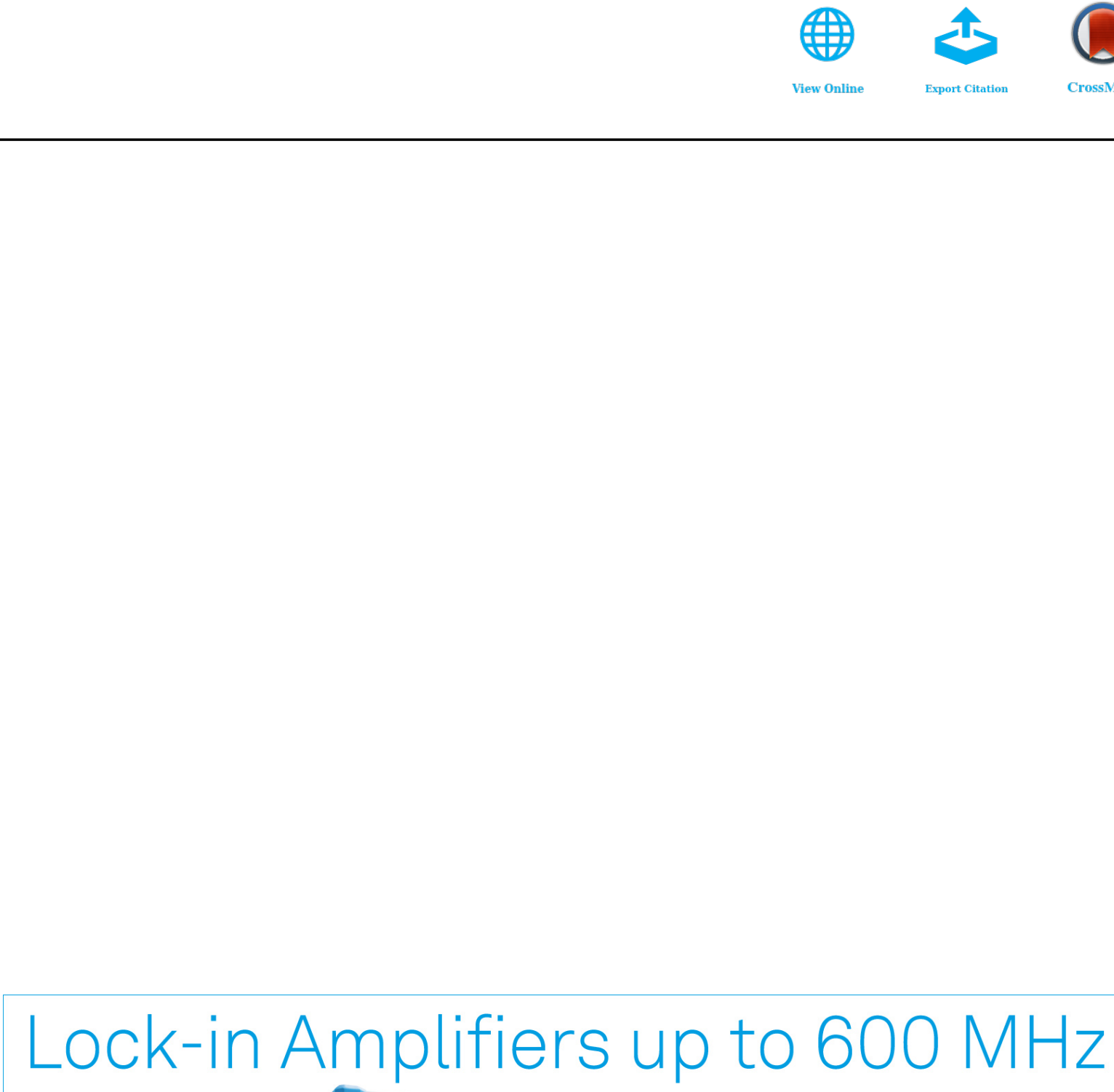

starting at $\$ 6,210$

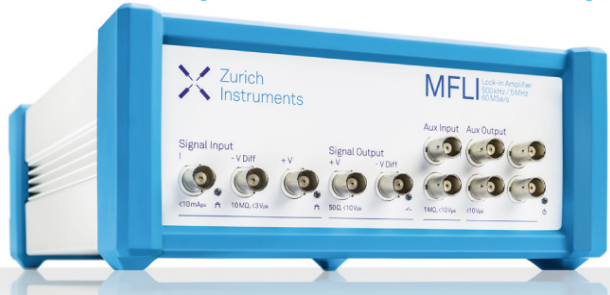

J. Chem. Phys. 150, 234508 (2019); https://doi.org/10.1063/1.5091682 (c) 2019 Author(s).
150,234508
Watch the Video 


\title{
Static and dynamic correlation lengths in supercooled polymers
}

\author{
Cite as: J. Chem. Phys. 150, 234508 (2019); doi: 10.1063/1.5091682 \\ Submitted: 4 February 2019 - Accepted: 31 May 2019 • \\ Published Online: 21 June 2019
}

Cristian Balbuena, ${ }^{\text {a) }}$ Melisa M. Gianetti, and Ezequiel R. Soulé ${ }^{b)}$

\begin{abstract}
AFFILIATIONS
Institute of Materials Science and Technology (INTEMA), University of Mar del Plata and National Research Council (CONICET), J. B. Justo 4302, 7600 Mar del Plata, Argentina

a) Electronic mail: cbalbuena@fi.mdp.edu.ar

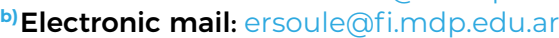

\begin{abstract}
A key point to understand the glass transition is the relationship between structural and dynamic behavior experienced by a glass former when it approaches $T_{g}$. In this work, the relaxation in a simple bead-spring polymer system in the supercooled regime near its glass transition temperature was investigated with molecular dynamic simulations. We develop a new manner to look at the dynamic length scales in a supercooled polymeric system, focusing on correlated motion of particles in an isoconfigurational ensemble (that is, associated with the structure), as measured by Pearson's correlation coefficient. We found that while the usual dynamic four-point correlation length deviates from the structural (mosaic or point-to-set) length scale at low temperatures, Pearson's length behaves similarly to the static length in the whole temperature range. The results lead to a consensus of similar scaling of structural and dynamical length scales, reinforcing the idea of the theories of Adam-Gibbs and random first order transition.
\end{abstract}

Published under license by AIP Publishing. https://doi.org/10.1063/1.5091682

\section{INTRODUCTION}

Glasses are deeply supercooled materials that behave as solids, but display no apparent molecular order such that from the molecular structure point of view, they look like liquids. They find use in many technological applications. In particular, many polymeric materials used in industrial products are glassy polymers. In order to control the viscoelastic and mechanical properties to improve the performance of these amorphous materials, understanding the behavior of static and dynamic length scales is expected to provide valuable insights in understanding this phenomenology. ${ }^{1-7}$ There are several theories that associate structural causes with dynamic behavior. In the Adam-Gibbs (AG) theory, for example, the large increase in relaxation times when the glass transition temperature $\left(T_{\mathrm{g}}\right)$ is approached is ascribed to the emergence of Cooperative Relaxation Regions (CRRs), which grow in size as the system approaches its $T_{g}{ }^{8}$ These CRRs represent clusters of particles that have a highly correlated movement. In a similar way that AG proposes the existence of CRR, the Random First Order Transition (RFOT) theory proposes, based on a statistical mechanical formulation at the mean-field level, a mosaic picture in which the liquid is divided into metastable regions with a characteristic size $\xi$ (the mosaic length). ${ }^{9,10}$ In this way, both AG and RFOT describe regions of correlated particles in the supercooled state that grow as the system approaches the $T_{g}$. This means that according to these two theories, the increase in relaxation time is caused by the growth of a purely static length scale, $\xi$, that represent some "hidden order" in the many-body free-energy landscape. In this sense, both theories describe a thermodynamic origin to explain the glass transition. Through large scale computer simulations, it can be shown that the most mobile particles can be further divided into groups of atoms or molecules that move cooperatively in a roughly stringlike fashion. ${ }^{11-13}$ The particles that present this dynamic behavior are identified as belonging to CRRs, and this describes the scenarios of the Adam-Gibbs and RFOT theory fairly well. ${ }^{14}$ There is evidence of an increase in the number of dynamically correlated particles as the temperature decreases. ${ }^{15}$ Moreover, many interesting studies in the last years trying to uncover some "hidden" structural order related to heterogeneous dynamics. For example, some results suggest that particles with low mobility tend to be found in certain locally preferred structures (LPS) or high local bond orientational order, and this tendency increases with supercooling. ${ }^{16-20}$ A striking result is 
that the relation between LPS and dynamical slowdown is highly system dependent. ${ }^{21}$ An alternative approach to those cited, is the point-to-set method ${ }^{22}$ which consists in measuring an overlap function in a region of mobile particles, surrounded by an environment of artificially frozen particles. With this technique, an order-agnostic correlation length can be defined, and it was found that it grows faster than simpler static lengths in various glass-forming liquids as $T_{g}$ is approached, supporting the RFOT scenario. ${ }^{22-26}$ Recent experimental studies reinforce these results. ${ }^{27}$ Another type of approach is given by the isoconfigurational ensemble method (ICEM) ideated by Widmer-Cooperet al. ${ }^{28}$ This technique consists in performing many molecular dynamic (MD) trajectories, with different initial velocities but the same initial positions. It allowed us to identify regions of high mobility and low mobility that are defined by the initial configuration, as well as the presence of correlations (between different trajectories of the ICE, measured through Pearson's correlation coefficient) in the displacement of neighboring particles. ${ }^{29-31}$ ICEM appears as a very useful tool to evidence the intimate relation between dynamics and the structure. In some models, localized soft modes appear to correlate strongly with propensity for motion at short time scale. ${ }^{32}$ One of the problems that arise when analyzing the supercooled state of glass-former is that the structural correlation lengths show a much smaller increase with the temperature than the dynamic length, where the latter increases more in the temporal scales of the simulation. ${ }^{6}$ This discrepancy found between the different correlation lengths is still a subject of debate and it is a key piece since it can reveal if the glass transition contains a thermodynamic origin. Within the idea that a thermodynamic origin exists, two reasons can be presented for the discrepancy between both correlation lengths. One possibility is that the dynamic behavior is nonmonotonic in the supercooled region, and in the time scale inaccessible from the simulation, both types of length scale take similar values, ${ }^{33,34}$ although this approach is also controversial. ${ }^{35,36}$ The other possibility is to find a dynamical length scale that increases weakly with supercooling, in a similar way to length scales based on structural measures. Recently, Dunleavy et al. ${ }^{37}$ proposed an alternative way to observe the increase in dynamic lengths through information theoretic quantities employing an isonconfigurational ensemble. They found similar behavior between structural and dynamic length scales considering a dynamic length scale obtained through correlated motion of the particles. In line with this, we introduce in this work a new approach to analyze the dynamic length scales that emerge in glass forming liquids of linear polymers, focusing on the tendency of the particles to be dynamically correlated through ICEM. ${ }^{38}$ We define a dynamic length that emerges not as a function of the particles mobilities, but rather it is based on the particle correlations, where these correlations involve relative movements. We also calculate the structural length scale with the PtS method. The results show that both magnitudes grow in a similar manner. This result leads to a consensus of similar scaling of structural and dynamical length scales, reinforcing the idea of the theories of $A G$ and RFOT.

\section{METHODS}

MD simulations of a polymer glass-forming system were performed in a wide range of temperatures and structural relaxation time. The polymer was represented through the bead-spring model, ${ }^{39}$ with fully flexible chain molecules. All monomers interact through the Lennard-Jones (LJ) potential,

$$
U\left(r_{i j}\right)=4 \epsilon\left[\left(\frac{\sigma}{r_{i j}}\right)^{12}-\left(\frac{\sigma}{r_{i j}}\right)^{6}\right],
$$

where $r_{i j}$ is the distance between the beads $i$ and $j, \sigma$ is the distance at which the interparticle potential is zero, and $\epsilon$ is the depth of the potential at the minimum. Both parameters take the value of 1 . $U\left(r_{i j}\right)$ is truncated for $r_{i j}$ greater than $2.0 \sigma$ with the long-tail correction applied. In addition, the bonded neighbors in a chain interact through the FENE bond potential,

$$
U_{\text {bond }}\left(r_{i j}\right)=-15 R_{0}^{2} \ln \left[1-\left(r_{i j} / R_{0}\right)^{2}\right],
$$

where $R_{0}=1.5 \sigma$ is the maximum length of the bond.

Simulations were implemented with the software LAMMPS ${ }^{40}$ with periodic boundary conditions. All values are reported in reduced LJ units. The time step for integration was $\mathrm{dt}=0.01 \mathrm{using}$ the Verlet velocity algorithm. Our results were based on systems of 2000 polymer chains containing 30 monomers each, except for the ICEM that worked with 133 chains. Full equilibration at each temperature was verified by the absence of drift in thermodynamic magnitudes and by the absence of aging. ${ }^{38}$ The NVE ensemble was used for production runs and for later production of the ICEM. At each studied temperature, 15 ICEs were generated, each one starting from equilibrated configurations obtained from independent trajectories. The ICE consisted of 500 trajectories.

\section{RESULTS AND DISCUSSION}

Since many different time scales are present in glassy relaxation, it is necessary to define a time scale to study the dynamic length scales. The relaxation of this model has been extensively studied in previous simulations. In Fig. 1(a), we show the behavior of the incoherent scattering function $F_{s}\left(q_{0}, t\right)$ at the wave vector $q_{0}$ (corresponding to the first peak of the structure factor). The structural relaxation time $(\tau)$ can be defined as the time at which $F_{s}(q, \tau)$ $=e^{-1}$. Figure 1(b) shows the relaxation times as a function of temperature. The behavior of $\tau(T)$ is well described by Vogel-FulcherTamman (VFT) expression: $\tau(T)=\tau_{0} \exp \left(A / T-T_{0}\right)$.

It can be noted that at high temperatures the behavior is Arrhenius type, with a single energy for the relaxation process. Below a certain temperature $T_{A}$, the system presents a non-Arrhenius behavior, and from small temperature changes, drastic changes occur in $\tau$. In this sense, the $T_{A}$ is presented as a reference temperature, which marks the change in behavior that occurs in the relaxation of the system, and for this model, $T_{A} \sim 1$ and $T_{0}=0.37$. The $T_{g}$ of this model, measured as the temperature at which a change in slope of the specific volume as a function of temperature is evidenced, is close to $0.45 .^{41}$ There is a general consensus that glass-forming liquids are dynamically heterogeneous, ${ }^{2,42,43}$ that is, they present a significant fraction of particles with extremely high or low mobility relative to the average, whose positions are spatially correlated. Dynamic heterogeneity may be interpreted through some structural origin, like the formation of CRRs or the aperiodic mosaic structures from RFOT theory. Glass-forming systems present 


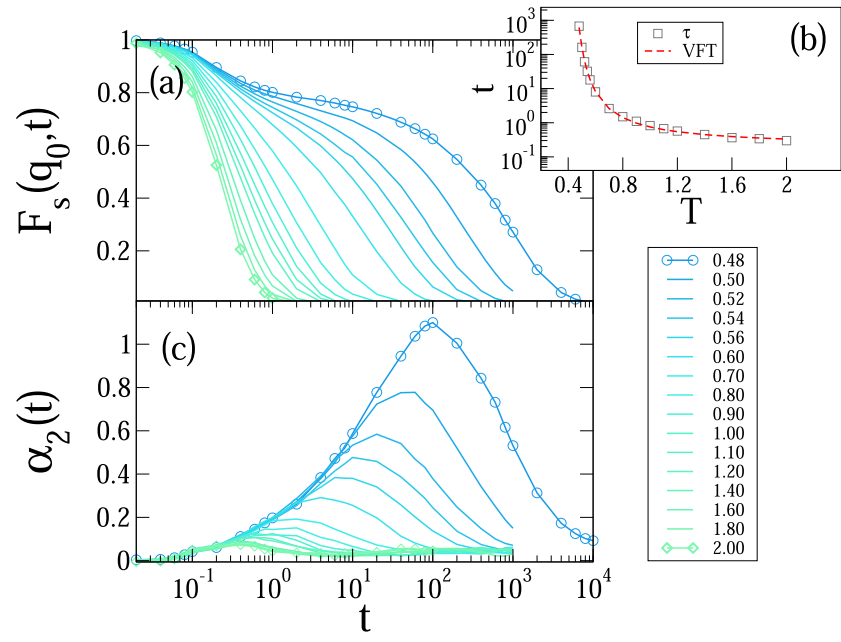

FIG. 1. (a) The behavior of the incoherent scattering function for the different temperatures at the slowest mode $q_{0}$. The relaxation time of the system is extracted from this function. (b) Relaxation times of the system as a function of temperature, and the dashed line corresponds to the VFT law. The temperature $T_{A}$ is the temperature at which the activation energy for the relaxation process changes (grows). (c) The behavior of the non-Gaussian parameter at the different temperatures. From this function, the times of maximum dynamic heterogeneity $\left(t^{*}\right)$ are obtained at the maximum of the function.

a characteristic time at which dynamically heterogeneity is maximum; this time is defined as $t^{*}$, and it can be extracted from the maximum of the non-Gaussian parameter $\left(\alpha_{2}\right)$. If dynamic heterogeneities are indeed related to CRRs, $t^{*}$ would be a reasonable time scale to observe the CRRs. Figure 1(c) shows the behavior of this function. It can be seen in this figure that when temperature decreases, $\alpha_{2}$ is larger, indicating that the heterogeneity increases, and also the maximum of the curves is observed at larger time, so $t^{*}$ increases. In order to quantify the increase in dynamic correlation lengths when the system approaches $T_{g}$, we propose to quantify the degree of correlation between monomers at $t^{*}$ through the ICEM. From our previous work, it can be shown that the highest correlation intensity is evidenced in $t^{*}{ }^{38}$ This method allows us to measure correlations in particle dynamics that are encoded in the initial configuration of the system. ${ }^{31}$ Since the correlations discussed here are measured in the isoconfigurational ensemble, we know that they have a structural origin and they must be caused by the initial particle configuration as this is the only thing in common between the different trajectories. The Pearson coefficient $\left(k_{i j}\right)$ is calculated on each ICE. ${ }^{38}$ This magnitude reflects the degree of dynamic correlation between the particles $i$ and $j$. The value of $k_{i j}^{*}$ at $t^{*}$, for two particles initially separated by a distance $r_{i j}=\left|r_{i}(0)-r_{j}(0)\right|$, is defined as

$$
k_{i j}^{*}\left(r_{i j}\right)=\frac{1}{S_{i}\left(t^{*}\right) S_{j}\left(t^{*}\right)} \sum_{w=1}^{N_{I C}} \psi_{i}\left(w, t^{*}\right) \psi_{j}\left(w, t^{*}\right),
$$

and the term $\psi_{i}\left(w, t^{*}\right)$ is given by

$$
\psi_{i}\left(w, t^{*}\right)=\mid\left(\mathbf{r}_{i}\left(w, t^{*}\right)-\mathbf{r}_{i}(0) \mid-\left\langle\Delta r_{i}\left(t^{*}\right)\right\rangle_{I C},\right.
$$

where $\left\langle\Delta r_{i}\left(t^{*}\right)\right\rangle_{\text {IC }}$ is the mean displacement at time $t^{*}$ in all the trajectories of the ICE for the particle $i$ (propensity), $S_{i}$ is the standard deviation of the propensity, $\psi_{i}(w, t)$ is the relative displacement of the monomer $i$ in a particular trajectory $w$ with respect to its propensity, and $r_{i}(0)$ is the position of the monomer $i$ in the initial configuration that generates the ICE. The coefficient $k_{i j}^{*}\left(r_{i j}\right)$ can take values between -1 and +1 and it is a measure of the correlation between displacement of two monomers, relative to the ICE average displacement (propensity) of each monomer. A negative correlation coefficient between two monomers implies that a large relative movement of one monomer is conditioned to a small relative movement of the other. A positive correlation implies that both monomers tend to present simultaneously large or small relative movement. A small value of the correlation coefficient implies that the relative movement of the monomers is independent of each other. In this work, the sum in $j$ in Eq. (3) is done for all the monomers of the system, whether they are bonded or not. In a previous work, we showed that there are no significant differences in the correlated dynamic behavior excluding monomers of the same chain in times relative to $t^{*}{ }^{38}$ To quantify the degree of global correlation at $t^{*}$ in the whole system for a certain distance $r$, we calculated the following quantity:

$$
K\left(r, t^{*}\right)=\frac{\sum_{i j}^{N} k_{i j}^{*}\left(r_{i j}\right) \delta\left(r-r_{i j}\right)}{\sum_{i j}^{N} \delta\left(r-r_{i j}\right)},
$$

where $\delta()$ denotes the Dirac delta function. So, the magnitude $K\left(r, t^{*}\right)$ reflects the global dynamic correlation between monomers at distance $r$. This global magnitude reflects correlated movements but in terms of relative movements of each particle, relative to its propensities for movement in the ICE. To quantify the correlation in terms of individual movements in relation with bulk dynamical behavior in a normal ensemble with NVT condition, that is, taking into account the displacement of a monomer with respect to the average displacement among all the particles, the following function is used:

$$
C_{\delta u}\left(r, t^{*}\right)=\frac{\sum_{i=1}^{N} \delta u_{i}\left(t^{*}\right) \sum_{j=1}^{N} \delta u_{j}\left(t^{*}\right) \delta\left(r-r_{i j}\right)}{\left\langle\delta u\left(t^{*}\right)^{2}\right\rangle \sum_{i, j} \delta\left(r-r_{i j}\right)},
$$

where

$$
\delta u_{i}\left(t^{*}\right)=\mid\left(\mathbf{r}_{i}\left(t^{*}\right)-\mathbf{r}_{i}(0) \mid-\left\langle\Delta r\left(t^{*}\right)\right\rangle .\right.
$$

The term $\left\langle\Delta r\left(t^{*}\right)\right\rangle$ is the average value of the displacement among all the particles and

$$
\left\langle\delta u(t)^{2}\right\rangle=\frac{1}{N} \sum_{w=1}^{N} \delta u_{i}(t)^{2} .
$$

The magnitude $C_{\delta u}\left(r, t^{*}\right)$ is similar to the standard four-point correlation function. ${ }^{44}$ The principal difference with $K\left(r, t^{*}\right)$ is that $C_{\delta u}\left(r, t^{*}\right)$ contemplates a correlated movement of the particles at $t^{*}$ but in relation to the average displacement of all particles, contrary to the case of $K\left(r, t^{*}\right)$ where the correlation of a monomer with its surroundings is relative to the tendency of its own 
movement (only possible through an ICE). Figure 2 shows the behavior of $K\left(r, t^{*}\right)$ and $C_{\delta u}\left(r, t^{*}\right)$ at different temperatures. It can be noted that the structure is affecting these dynamic correlations at $t^{*}$ since the behavior of the curves oscillates similarly to the radial distribution function $g(r){ }^{38}$ It is observed that correlation increases when the temperature decreases and the correlation is significant at larger distances. In the inset of Fig. 2, the behavior of $C_{\delta u}\left(r, t^{*}\right)$ (dashed lines) is compared to $K\left(r, t^{*}\right)$ (continuous lines) for three different temperatures. We found that at low temperatures, the difference between both functions increases with the distance, while at high temperatures, both magnitudes are coincident. In our previous work, we show that this magnitude of correlation presents its greatest intensity at time $t^{*}$. In comparison with the structural relaxation time, where the correlations decay to very low values, a $t^{*}$ correlation extends to greater distances than the first neighbors. This is the reason why in this case we analyze the correlation lengths at this characteristic time. A correlation length can be extracted from the decay of $K\left(r, t^{*}\right)$ and $C_{\delta u}\left(r, t^{*}\right)$ as a function of the distance, similar to other works: ${ }^{44-47}$ An exponential decay, exp - $\left(r^{\prime} / \xi\right)$, is fitted to $K\left(r^{\prime}, t^{*}\right)$ and $C_{\delta u}\left(r, t^{*}\right)$, where $r^{\prime}$ denotes the local maxima of each function; and $\xi$ is the correlation length, which is different for each function. Thereby, $\xi_{K}$ reflects the size of regions formed by dynamically connected particles, while $\xi_{\delta u}$ reflects the size of regions with particles of similar mobility. It should be kept in mind that the calculation of the four-point dynamic correlation length, $C_{\delta u}\left(r, t^{*}\right)$, in this way, results in somewhat smaller values than calculating it by the reciprocal space. ${ }^{44,48}$ Figure 3 shows the calculated correlation lengths. A similar behavior in both dynamic lengths is observed at high temperatures and they both increase proportionally to each other, but at low temperatures, $\xi_{\delta u}$ increases faster than $\xi_{K}$ and the

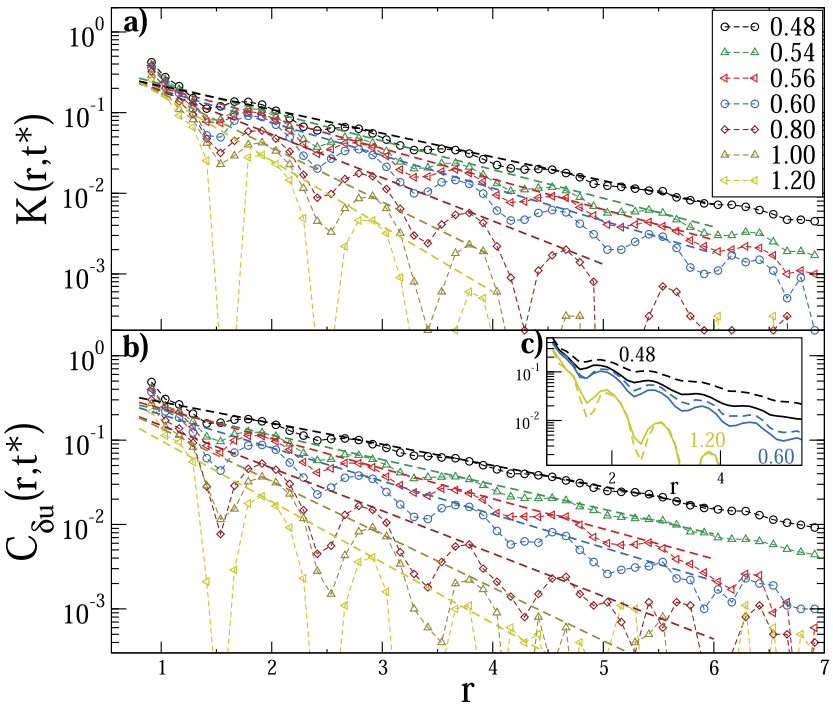

FIG. 2. (a) The behavior of $K\left(r, t^{*}\right)$ at the different temperatures. The dashed lines correspond to the linear fits that describe the linear expression $K\left(r, t^{*}\right) \sim \exp$ - $\left(r / \xi_{K}\right)$ and from which the correlation lengths can be obtained. (b) The behavior for $C_{\delta u}\left(r, t^{*}\right)$. (c) Comparison between $K\left(r, t^{*}\right)$ (continuous lines) and $C_{\delta u}\left(r, t^{*}\right)$ (dashed lines) for three different temperatures.

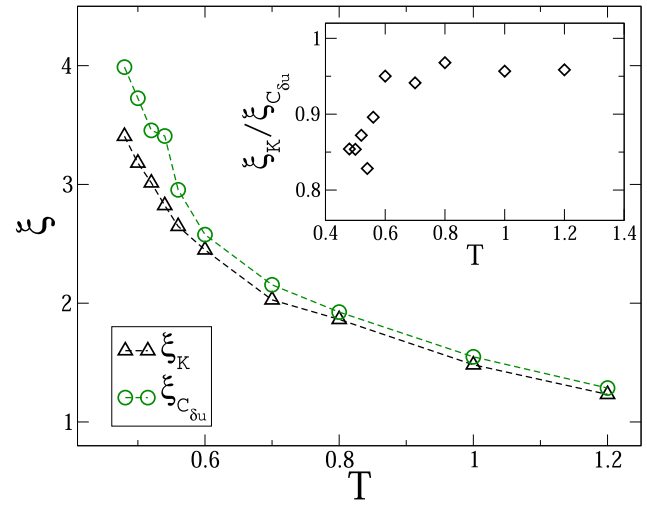

FIG. 3. Dynamic correlation length obtained from the behavior of $K\left(r, t^{*}\right)$ and $C_{\delta u}\left(r, t^{*}\right)$ at the different temperatures studied. Inset: the relationship between both lengths is shown. It can be seen how, at approximately $T=0.70$, a change in behavior occurs in which the proportionality between both magnitudes is lost for lower temperatures

proportionality is broken (this is explicitly shown in the inset of Fig. 3). This discrepancy that arises at low temperatures is another indication that the dynamic lengths in terms of the absolute displacements of the monomers depart from the structural behavior, showing a greater increase. ${ }^{6,33,34,49}$ On the other hand, the relative displacements, $\xi_{K}$, show a softer increase, as found in some structural lengths. ${ }^{18,19}$ The decoupling of both length at about $\mathrm{T}=0.7$ could be an indication of a change in the relaxation mechanism, and this will be discussed in more detail later. We remark that despite the observed discrepancies, these types of correlation lengths increase continuously on supercooling toward the glass transition. In order to compare these dynamic correlation lengths with a structural one, we calculated the static correlation length based on the PtS method, following the protocol of Ref. 22. This method allows us to calculate a nontrivial structural length scale $\left(\xi_{P t S}\right)$, which measures the distance over which particles are self-consistently pinned to other particles. For each temperature, 18 bulk equilibrium configurations were generated at a desired temperature, and then, cavities were constructed by freezing the particles outside a sphere of radius $R$. Figure 4 shows a graphical representation of the spherical cavity of the center that contains mobile monomers (large particles in colors, where each color represents different polymer chains) and monomers outside the cavity that are immobile (small particles in blue color). The center of the cavity is partitioned into many small cubic boxes of length $l$, generating a subvolume $v$. The overlap function is then defined as

$$
q_{c}(R)=\frac{1}{l^{3} N_{b}} \sum_{i \in v}\left\langle n_{i}\left(t_{0}\right) n_{i}\left(t_{0}+\infty\right)\right\rangle
$$

The sum runs over all the boxes at the center of the sphere $\left(N_{b}\right)$, and $\langle\cdots\rangle$ represents the averaging over different realizations of the frozen boundary. The function is normalized in such a way that two identical configurations have overlap equal to unity and two uncorrelated random configurations have overlap close to $q_{0}=l^{3}$. We used replica exchange molecular dynamics (REMD) ${ }^{50}$ to sample the equilibrium thermodynamic properties of the system in the mobile 


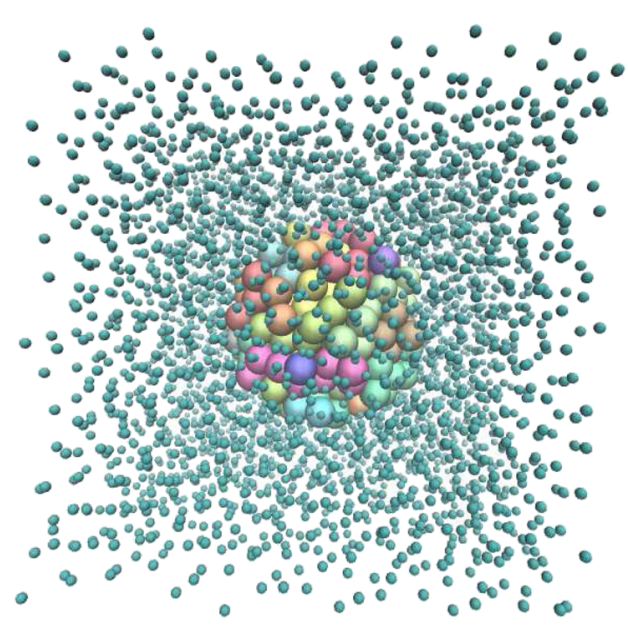

FIG. 4. Representation of the spherical cavity that contains mobile particles and the monomers outside the cavity that are immobile monomers (small blue particles).

cavities of PtS. In our case, the REMD consisted of 16 noninteracting replicas of the same system at different temperatures $T_{i}\left(T_{1}, \ldots\right.$, $T_{16}$ ), where $T_{1}$ is the temperature of interest. The difference in temperature between two neighboring replicas was 0.01 , which gives a good overlap of the potential energy distributions. Each attempted swap of neighbor temperatures is either accepted or rejected based on a Boltzmann-weighted Metropolis criterion and the exchange between different replica was done every $0.025 \tau\left(T_{1}\right)$ (the relaxation time at the low temperature, the target temperature). Then, each replica is run independently and simultaneously in a canonical ensemble for the total time of $10-100 \tau\left(T_{1}\right)$, depending on the temperature. This procedure was employed at each studied temperature (different $T_{1}$ ) with 18 independent configurations. The acceptance ratios of exchange between replicas ranged around 0.3 in the different experiments. The result of this swapping between different temperatures allowed us to jump across the energy barriers of the system to avoid the two barriers imposed in the system, first the natural barrier, the basin transition from the supercooled state, and second the artificial barrier, the $\mathrm{PtS}$ restriction conditions. By means of this procedure, it is possible to equilibrate the subsystem in the cavity and thus obtain equilibrium values for the overlapping function. A study that can be performed to check the correct balance within the cavity is the $\beta$ initial condition (BIC) test proposed in Refs. 51 and 52, where a random configuration is generated within the cavity (beta configuration) and then the corresponding temporal evolution is made starting from this configuration. Then, the same is done but starting from the initial balanced configuration (alpha configuration). If both cases converge to the same value of the overlap function at long enough times, that is considered to be the equilibrium value. Figure 5 shows the results of performing the BIC test for some of the particular states studied. It can be noted that in all cases both initial configurations type converge to the same value in the overlap function. Figure 6 shows the behavior of the overlap function $q(R)-q_{0}$ at different temperatures. It can be noted that as temperature decreases, the decay becomes slower. In order to extract a correlation length $\xi_{P t S}$ from the spatial decay of $q(R)$, a generalized compressed exponential of the form $q_{c}(r)-q_{0}$ $=\Omega \exp \left[-\left((R-a) / \xi_{P t S}\right)^{\zeta}\right]$ was fitted to the data. We fixed the value of $a=1$ and $0.55<\Omega<0.65$, in a similar manner to the work of Hocky et al., ${ }^{24}$ leading to values for $\xi$ with much smaller statistical variance. The dashed lines in Fig. 6 show the fits with this expression. Figure 7(a) shows the relation between both dynamic correlation lengths and the static correlation length, $\xi_{P t s}$. It has to

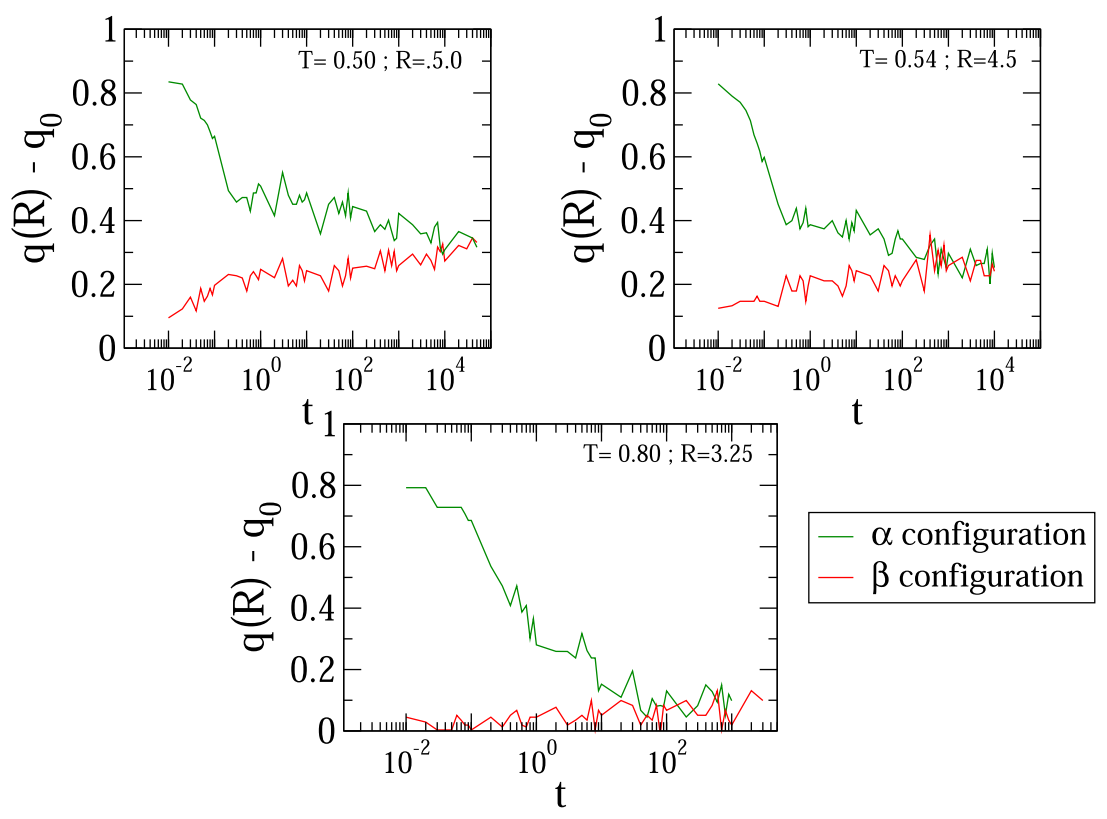

FIG. 5. BIC test for some of the temperatures and $\mathrm{R}$ studied, as indicated in each figure. The $\alpha$ and $\beta$ configurations reach the same asymptotic equilibrium value of $q(R)$ for large times. 


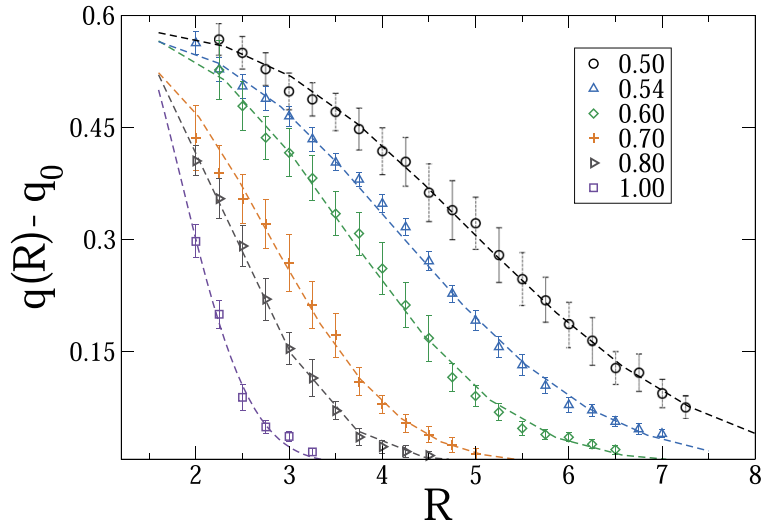

FIG. 6. PtS overlap function calculated at different temperatures. Dashed lines follow the expression $q_{c}(r)-q_{0}=\Omega \exp \left[-\left((R-1) / \xi_{\text {PtS }}\right)^{\zeta}\right]$ and are fitted to the calculated values at each temperature.

be noted that $\xi_{\text {PtS }}$ presents a larger increase with decreasing temperature, becoming large in absolute value, which is not observed in nonpolymeric systems studied by this method. This is probably due to the fact that in a polymeric system where the PtS is applied, there
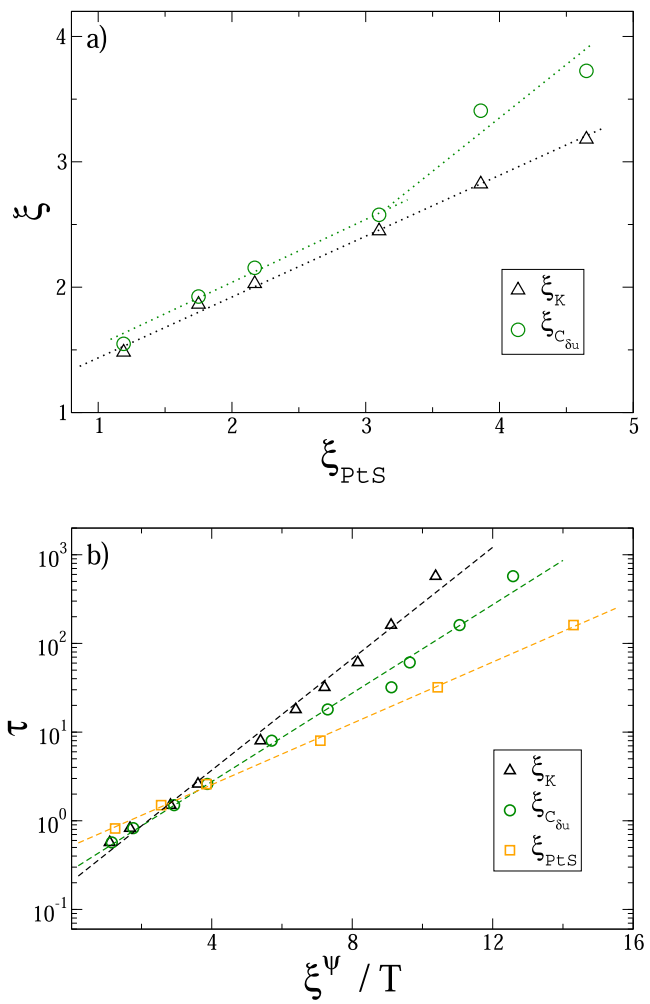

FIG. 7. (a) The relation between the dynamic correlation lengths $\left(\xi_{\delta u}\right.$ and $\left.\xi_{K}\right)$ and the static correlation length $\xi_{\text {pts }}$. The dashed lines are guide to the eye to highlight the behavior of both magnitudes. (b) The behavior of $\xi^{\psi}(T)$ following the expression of RFOT for the $\xi_{P t S}, \xi_{\delta u}$, and $\xi_{K}$, the values of $\psi$ are $1.28,1.30$, and 1.31 , respectively. are certain polymer chains that have mobile monomers $\left(r_{i}<R\right)$ and immobile $\left(r_{i}>R\right)$, which result in a double confinement in the mobile monomers. This affects the behavior of $q_{c}(R)$ and the consequent $\xi_{\text {PtS }}$. For this reason, the correlation lengths found for a connected system like polymers may be larger than other systems with similar intermolecular forces but without intramolecular connectivity. This connectivity in polymer systems causes that some properties differ from the typical glass-forming systems ${ }^{53}$ and a direct comparison of the absolute values of correlation lengths might then be deceiving. But as the relative dependence of one length with respect to the other is considered, a few interesting observations can be made. First, the 4 point correlation length, $C_{\delta u}$, as shown before, is proportional to $\xi_{K}$ at high temperatures, and this proportionality breaks down at about $\mathrm{T}=0.7$. In a similar way, it is found that $C_{\delta u}$ has a linear correlation with $\xi_{P t S}$, and this linearity is lost at this intermediate supercooling temperature. This behavior is usually found in glass formers, where the dynamic length extracted from four-point correlation functions is observed to grow to a greater extent than the structural length. ${ }^{4}$ On the other hand, $\xi_{K}$ shows a linear relation with $\xi_{P t S}$ in the whole temperature range studied. This seems to indicate that while correlations of the absolute displacements decorrelate from structural lengths at some intermediate degree of supercooling, correlations in terms of relative displacements can be described in terms of a structural length in the whole temperature range. Although this analysis is far from being the complete picture of the underlying physical mechanisms, it does seem to indicate that $\xi_{K}$ and $\xi_{\text {PtS }}$ correlation lengths are indeed related and this alternative calculation of a dynamic length can be the missing link to relate structure and dynamic in glass formers.

Finally, Fig. 7(b) shows the scaling of $\tau$ with $\xi_{P t S}, \xi_{\delta u}$, and $\xi_{K}$, as given by RFOT theory. For each curve, we obtain the best fit for the exponent $\psi$, where the values around 1.3 obtained are similar to those reported in different types of systems and according to the theory. 9,14,22,24 This indicates that RFOT describes the relaxation time in terms of correlation lengths fairly well, and that different correlation lengths, calculated from static or dynamic correlations, could be used as the characteristic "mosaic" size. However, and as previously discussed, only the behavior between $\xi_{K}$ and $\xi_{P t S}$ seems to indicate a correlation between them for the entire temperature range. It would be very interesting to extend this analysis to lower temperatures (larger lengths), but this would require larger simulation domains (which has to be significantly larger than the correlation length), and much larger time scales, not feasible with current computation capabilities.

\section{CONCLUSION}

In summary, we analyzed the increase in relaxation times and correlation lengths in a polymeric system through dynamics and structural analysis. The growth of a structural length was analyzed by the PtS using the replicate exchange method and two dynamic lengths were considered. The first one was the usual length defining a mobility correlation, calculated from the standard four-point correlation function. The second one was quantified through a statistical study within an isoconfigurational ensemble, defining the dynamic correlations between the particles by means of Pearson's correlation coefficient. It was shown that that there is a linear 
relation between $C_{\delta u}$ and $\xi_{P t S}$ at high temperatures, but this linearity is lost at an intermediate supercooling temperature. This is consistent with other studies where it is observed that the correlation lengths calculated from four-point correlation functions grow faster than the structural lengths with decreasing temperature for large supercooling. On the other hand, $\xi_{K}$ shows a linear relation with $\xi_{P t S}$ in the whole temperature range studied. This points out to an alternative way of defining dynamic lengths, through the ICEM, which could be useful to resolve the apparent observed discrepancies between structural and dynamic length scales in supercooled liquids.

\section{ACKNOWLEDGMENTS}

We thank T. Grigera for comments in the early stages of this work. This work was supported by grants from Consejo Nacional de Investigaciones Científicas y Técnicas (CONICET, Argentina) and Universidad Nacional de Mar del Plata. C.B. and E.R.S. are research fellows of CONICET, and M.M.G. thanks CONICET for a fellowship. We used Mendieta Cluster from CCAD-UNC and Toko Cluster from FCEN-UNCuyo, which are part of SNCAD-MinCyT, Argentina.

\section{REFERENCES}

${ }^{1}$ P. G. Debenedetti and F. H. Stillinger, Nature 410, 259 (2001).

${ }^{2}$ L. Berthier and G. Biroli, Rev. Mod. Phys. 83, 587 (2011).

${ }^{3}$ A. Cavagna, Phys. Rep. 476, 51 (2009).

${ }^{4}$ P. Charbonneau and G. Tarjus, Phys. Rev. E 87, 042305 (2013).

${ }^{5}$ C. P. Royall and S. R. Williams, "The role of local structure in dynamical arrest," Phys. Rep. 560, 1 (2015).

${ }^{6}$ S. Karmakar, C. Dasgupta, and S. Sastry, Rep. Prog. Phys. 79, 016601 (2016).

${ }^{7}$ L. Berthier, G. Biroli, J.-P. Bouchaud, and G. Tarjus, J. Chem. Phys. 150, 094501 (2019).

${ }^{8}$ G. Adam and J. H. Gibbs, J. Chem. Phys. 43, 139 (1965).

${ }^{9}$ T. R. Kirkpatrick, D. Thirumalai, and P. G. Wolynes, Phys. Rev. A 40, 1045 (1989).

${ }^{10}$ J.-P. Bouchaud and G. Biroli, J. Chem. Phys. 121, 7347 (2004).

${ }^{11}$ C. Donati, J. F. Douglas, W. Kob, S. J. Plimpton, P. H. Poole, and S. C. Glotzer, Phys. Rev. Lett. 80, 2338 (1998).

${ }^{12}$ M. Aichele, Y. Gebremichael, F. W. Starr, J. Baschnagel, and S. C. Glotzer, J. Chem. Phys. 119, 5290 (2003).

${ }^{13}$ Y. Gebremichael, M. Vogel, and S. C. Glotzer, J. Chem. Phys. 120, 4415 (2004).

${ }^{14}$ F. W. Starr, J. F. Douglas, and S. Sastry, J. Chem. Phys. 138, 12A541 (2013).

${ }^{15}$ T. Bauer, P. Lunkenheimer, and A. Loidl, Phys. Rev. Lett. 111, 225702 (2013).

${ }^{16}$ D. Coslovich and G. Pastore, J. Chem. Phys. 127, 124504 (2007).

${ }^{17}$ J. Ding, Y.-Q. Cheng, H. Sheng, and E. Ma, Phys. Rev. B 85, 060201 (2012).

${ }^{18}$ C. Patrick Royall, S. R. Williams, T. Ohtsuka, and H. Tanaka, Nat. Mater. 7, 556 (2008).
${ }^{19}$ H. Tanaka, T. Kawasaki, H. Shintani, and K. Watanabe, Nat. Mater. 9, 324 (2010).

${ }^{20}$ J. E. Hallett, F. Turci, and C. P. Royall, Nat. Commun. 9, 3272 (2018).

${ }^{21}$ G. M. Hocky, D. Coslovich, A. Ikeda, and D. R. Reichman, Phys. Rev. Lett. 113, 157801 (2014).

${ }^{22}$ G. Biroli, J.-P. Bouchaud, A. Cavagna, T. S. Grigera, and P. Verrocchio, Nat. Phys. 4, 771 (2008).

${ }^{23}$ L. Berthier and W. Kob, Phys. Rev. E 85, 011102 (2012).

${ }^{24}$ G. M. Hocky, T. E. Markland, and D. R. Reichman, Phys. Rev. Lett. 108, 225506 (2012).

${ }^{25}$ G. Gradenigo, R. Trozzo, A. Cavagna, T. S. Grigera, and P. Verrocchio, J. Chem. Phys. 138, 12 A509 (2013).

${ }^{26}$ G. Biroli and C. Cammarota, Phys. Rev. X 7, 011011 (2017).

${ }^{27}$ S. Albert, T. Bauer, M. Michl, G. Biroli, J.-P. Bouchaud, A. Loidl, P. Lunkenheimer, R. Tourbot, C. Wiertel-Gasquet, and F. Ladieu, Science 352, 1308 (2016).

${ }^{28}$ A. Widmer-Cooper, P. Harrowell, and H. Fynewever, Phys. Rev. Lett. 93, 135701 (2004).

${ }^{29}$ A. Widmer-Cooper and P. Harrowell, J. Phys.: Condens. Matter 17, S4025 (2005).

${ }^{30}$ A. Widmer-Cooper and P. Harrowell, Phys. Rev. Lett. 96, 185701 (2006).

${ }^{31}$ A. Widmer-Cooper and P. Harrowell, J. Chem. Phys. 126, 154503 (2007).

${ }^{32}$ A. Widmer-Cooper, H. Perry, P. Harrowell, and D. R. Reichman, Nat. Phys. 4, 711-715 (2008).

${ }^{33}$ W. Kob, S. Roldán-Vargas, and L. Berthier, Nat. Phys. 8, 164 (2011).

${ }^{34}$ K. Hima Nagamanasa, S. Gokhale, A. K. Sood, and R. Ganapathy, Nat. Phys. 11, 403 (2015).

${ }^{35}$ E. Flenner and G. Szamel, Nat. Phys. 8, 696 (2012).

${ }^{36}$ B. Mei, Y. Lu, L. An, H. Li, and L. Wang, Phys. Rev. E 95, 050601 (2017).

${ }^{37}$ A. J. Dunleavy, K. Wiesner, R. Yamamoto, and C. P. Royall, Nat. Commun. 6, 6089 (2015).

${ }^{38}$ C. Balbuena, M. M. Gianetti, and E. R. Soulé, J. Chem. Phys. 149, 094506 (2018).

${ }^{39}$ G. S. Grest and K. Kremer, Phys. Rev. A 33, 3628 (1986).

${ }^{40}$ S. Plimpton, J. Comput. Phys. 117, 1 (1995).

${ }^{41}$ T. Yamazaki, J. Phys. Chem. B 118, 14687 (2014).

${ }^{42}$ B. Rijal, L. Delbreilh, and A. Saiter, Macromolecules 48, 8219 (2015).

${ }^{43}$ E. Flenner and G. Szamel, J. Chem. Phys. 138, 12A523 (2013).

${ }^{44}$ N. Lačević, F. W. Starr, T. B. Schrøder, V. N. Novikov, and S. C. Glotzer, Phys. Rev. E 66, 030101 (2002).

${ }^{45}$ B. Doliwa and A. Heuer, Phys. Rev. E 61, 6898 (2000).

${ }^{46}$ E. R. Weeks, J. C. Crocker, and D. A. Weitz, J. Phys.: Condens. Matter 19, 205131 (2007).

${ }^{47}$ F. Puosi and D. Leporini, J. Chem. Phys. 136, 164901 (2012).

${ }^{48}$ N. Lačevic, F. W. Starr, T. B. Schröder, and S. C. Glotzer, J. Chem. Phys. 119, 7372 (2003).

${ }^{49}$ D. Ganapathi, K. H. Nagamanasa, A. K. Sood, and R. Ganapathy, Nat. Commun. 9, 397 (2018).

${ }^{50}$ K. Hukushima and K. Nemoto, J. Phys. Soc. Jpn. 65, 1604 (1996).

${ }^{51}$ A. Cavagna, T. S. Grigera, and P. Verrocchio, J. Chem. Phys. 136, 204502 (2012).

${ }^{52}$ L. Berthier, P. Charbonneau, and S. Yaida, J. Chem. Phys. 144, 024501 (2016).

${ }^{53}$ J. Colmenero, J. Phys.: Condens. Matter 27, 103101 (2015). 